\title{
The heterogeneity of lung perfusion patterns in SPECT/CT during COVID-19: not only embolism
}

\author{
Lavinia Monaco $^{1}$ (1) $\cdot$ Cinzia Crivellaro $^{2} \cdot$ Massimo Cressoni $^{3} \cdot$ Giuseppe Foti $^{3} \cdot$ Claudio Landoni $^{1,2} \cdot$ Cristina Messa $^{1}$. \\ Luca Guerra ${ }^{1,2}$
}

Received: 6 April 2021 / Accepted: 22 April 2021 / Published online: 5 May 2021

(C) The Author(s), under exclusive licence to Springer-Verlag GmbH Germany, part of Springer Nature 2021

\section{Dear Sir,}

We read with great interest the manuscript of Das et al. about the role of perfusion SPECT/CT in the detection of pulmonary embolism (PE) in COVID-19 patients with moderate/high probability of PE [1]. Although this kind of studies generally employs a combined ventilation/perfusion (V/Q) approach to assess the presence of pulmonary embolism, during the COVID-19 pandemic safety concerns led to discordant opinions on the "routine" V/Q protocols [2] and only recently some societies suggested the possibility to integrate ventilation in specific and selected circumstances [3]. However, the experience of Das et al. well demonstrated how even the perfusion alone could play a possible role in the study of these complex and delicate cases [1]. Indeed, one of the main complications of COVID-19 is related to diffuse and localized thromboembolic events, particularly involving the pulmonary microcirculation, and functional perfusion studies coupled with CT images (SPECT/CT) can significantly help in the prompt detection of these cases. Moreover, it can eventually shed light on the peculiar phenotype described in the routine practice, characterized by severe hypoxemia with relatively preserved lungs at $\mathrm{CT}$, the socalled happy hypoxic state [4]. The previous employment of other perfusion techniques (e.g., dual-energy CT) showed peculiar vasodilation phenomena without embolic defects in

This article is part of the Topical Collection on Letter to the Editor

Cinzia Crivellaro

cinzia.crivellaro@unimib.it

1 School of Medicine and Surgery, University of Milano Bicocca, Monza, Italy

2 Nuclear Medicine, San Gerardo Hospital, University of Milano Bicocca, Monza, Italy

3 Department of Medicine and Surgery, Emergency and Intensive Care, ASST Monza, University of Milano Bicocca, Monza, Italy consolidated lungs during COVID-19 pneumonia [5]. This stressed on one hand the need for functional studies, suggesting possible interpretative keys for the already mentioned "happy hypoxic" patients, and on the other hand supporting the putative role of more safe and "infection-free" procedures, as the perfusion-only studies. In our experience of primary center facing the different phases of the pandemic in Italy, the Nuclear Medicine Unit of ASST Monza approached this urgent issue by the employment of perfusion SPECT/CT with 99mTc-macroaggregated albumin (MAA), offering the opportunity to measure the regional lung perfusion without aerosolization risks. Interestingly, in our series, two anecdotal cases demonstrated segmental perfusion defects with normal parenchyma at CT images (Fig. 1, panel a), eventually associated with focal areas of increased perfusion in consolidated and hypoventilated lung regions (Fig. 1, panel b). These functional and morphological modifications could help in the detection of suspected pulmonary embolisms, as well as in the definition of functional and radiological modifications that can partly explain the happy hypoxic phenomenon. Finally, SPECT/CT findings confirm the evidence of an increased perfusion around areas of lung consolidation [5], contributing to depicting the great heterogeneity of functional imaging patterns in COVID-19 pneumonia [6], that partly explain the "atypical" presentations of these specific cases of acute respiratory distress syndromes (ARDS). These observations stress the putative role of perfusion SPECT/CT in shedding light on the physio-pathogenesis of microvascular dysfunction in COVID-19, eventually helping in the detection of patients with worse outcome.

Author contribution All the authors contributed to the study conception and design. Material preparation, data collection, and analysis were performed by Lavinia Monaco, Cinzia Crivellaro, Massimo Cressoni, Giuseppe Foti, Claudio Landoni, Cristina Messa, and Luca Guerra. The first draft of the manuscript was written by Lavinia Monaco and all the authors commented on previous versions of the manuscript. All the authors read and approved the final manuscript. 
Fig. 1 Axial MAA-lung perfusion SPECT/TC. A 57-year-old male patient admitted to our hospital with ARDS due to bilateral SARS-CoV-2 pneumonia. MAAlung perfusion SPECT/TC confirmed the pulmonary embolism (elevated D-dimer and clinical symptoms), showing segmental baso-lateral perfusion defect of the right lower lobe, that was normally aerated at CT images (asterisk; panel a). In the second patient, a 48-year-old male admitted for the same reasons, SPECT/CT lung perfusion showed an increased perfusion in consolidated or hypoventilated lung regions compared to the normally inflated ones (panel b)

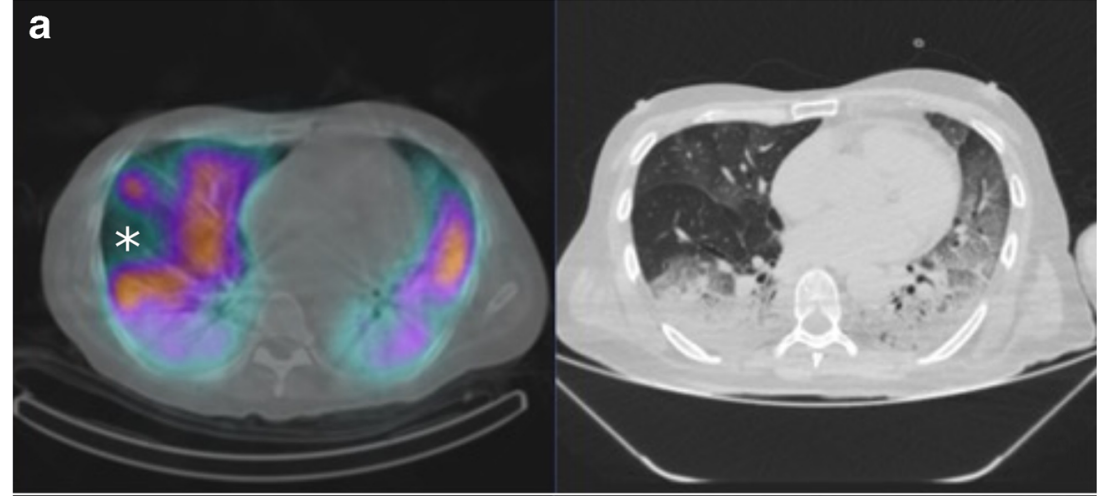

b

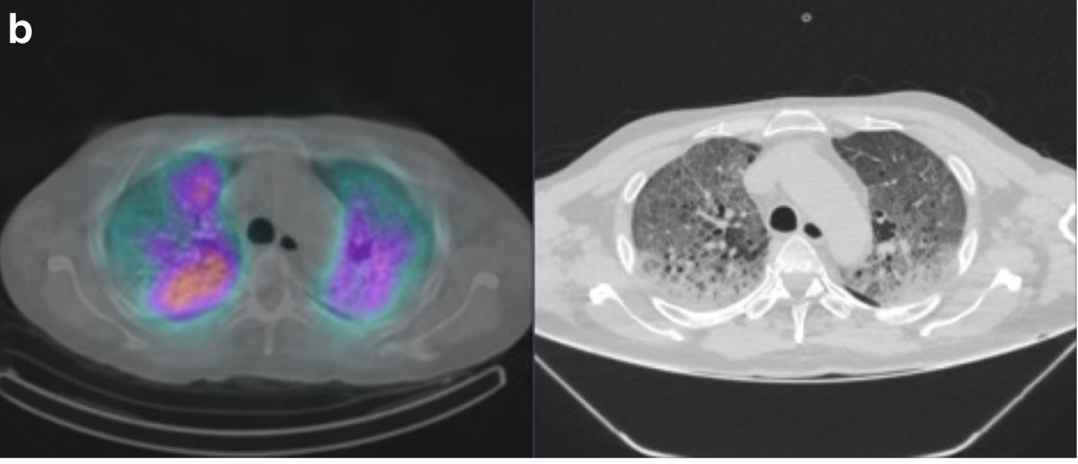

Availability of data and material Not applicable

Code availability Not applicable

\section{Declarations}

Ethics approval All the procedures performed in studies involving human participants were in accordance with the ethical standards of the institutional and/or national research committee and with the 1964 Helsinki Declaration and its later amendments or comparable ethical standards.

Consent to participate and for publication The article contains anonymized radiological images, not requiring a consent as per journal guidelines.

Conflict of interest The authors declare no competing interests.

\section{References}

1. Das JP, Yeh R, Schöder H. Clinical utility of perfusion (Q)-singlephoton emission computed tomography (SPECT)/CT for diagnosing pulmonary embolus (PE) in COVID-19 patients with a moderate to high pre-test probability of PE. Eur J Nucl Med Mol Imaging [Internet]. 2020; Available from: https://doi.org/10.1007/s00259020-05043-y

2. International Atomic Energy Agency. COVID-19 pandemic: technical guidance for nuclear medicine departments. 2020. https://www. iaea.org/publications/14733/covid-19-pandemic-technicalguidance-for-nuclear-medicine-departments. Accessed 1 Apr 2021

3. SNMMI Updates Statement on COVID-19, Ventilation/Perfusion (V/Q) Lung Studies [Internet]. [cited 2021 Mar 28]. Available from: https://www.appliedradiology.com/articles/snmmi-updatesstatement-on-covid-19-ventilation-perfusion-v-q-lung-studies.

4. Dhont S, Derom E, Van Braeckel E, Depuydt P, Lambrecht BN. The pathophysiology of "happy" hypoxemia in COVID-19. Respir Res. 2020;21:198

5. Lang M, Som A, Mendoza DP, Flores EJ, Reid N, Carey D, et al. Hypoxaemia related to COVID-19: vascular and perfusion abnormalities on dual-energy CT. Lancet Infect Dis. 2020;20:1365-6.

6. Cobes N, Guernou M, Lussato D, Queneau M, Songy B, Bonardel $\mathrm{G}$, et al. Ventilation/perfusion SPECT/CT findings in different lung lesions associated with COVID-19: a case series. Eur J Nucl Med Mol Imaging. 2020;47:2453-60.

Publisher's note Springer Nature remains neutral with regard to jurisdictional claims in published maps and institutional affiliations. 